\title{
Comparisons of Quality Ratings for Music by Cochlear Implant and Hearing Aid Users
}

\author{
Valerie Looi, Hugh McDermott, Colette McKay, and Louise Hickson
}

\begin{abstract}
Objectives: This study aimed to compare the quality ratings by cochlear implant $(\mathrm{CI})$ and hearing aid (HA) users in response to musical sounds.

Design: The ratings of 15 experienced adult Nucleus CI users $\equiv$ ig the ACE or SPEAK strategy) were compared the of 15 experienced adult HA users who met the audiological criteria for implantation. Additionally, nine subjects on the waiting list (WL) for an implant were tested before and after implantation. Three types of musical stimuli were used: single instruments, solo instruments with background accompaniment, and ensembles. For each of these categories, 12 different instruments or ensembles were presented four times each. Subjects were asked to provide a rating out of 10 according to how pleasant each extract sounded, with 10 being "very pleasant."
\end{abstract}

Results: For the WL subjects, ratings provided after implantation were significantly higher than their preimplant ratings obtained when using HAs $(p=$ 0.026). This was consistent with a trend observed from the experienced CI and HA groups, whereby the CI group provided higher ratings than the HA group for all three subtests, although the difference was not statistically significant. For all groups, single-instrument stimuli received significantly higher ratings than those involving multiple instruments (CI and HA subjects: $p<0.001$; WL subjects: $p=$ 0.034). With this research being part of a larger study in which identification testing of these stimuli had previously been conducted, significant correlations were also obtained between the subjects' ability to identify musical stimuli and the corresponding quality ratings $(\mathrm{CI}$ : rho $=0.325, p=0.029$; HA: $r h o=0.491, p=0.001)$.

Conclusions: The findings of this study suggest that although neither device enables highly satisfactory music appreciation, the CI users judged music to sound more pleasant than the HA users (who had similar levels of hearing impairment). Also, all subject groups appraised music that involved multiple

Department of Otolaryngology, The University of Melbourne, Melbourne, Australia (V.L., H.M., C.M.); Cooperative Research Centre for Cochlear Implant and Hearing Aid Innovation, $\equiv$ Melbourne, Australia (V.L.); School of Health and Life Scie $-\nabla$, Aston University, Birmingham, United Kingdom (C.M.); Division of Audiology, School of Health and Rehabilitation Sciences, The University of Queensland, $\equiv$ enstand, Australia (L.H.); and Department of Communice Disorders, The University of Canterbury, Christchurch, New Zealand (V.L.). instruments to sound less pleasant, on average, than music played by single instruments.

(Ear \& Hearing 2007;28;1-•)

The broadening of cochlear implant (CI) selection criteria has resulted not only in more patients being implanted but also in higher expectations of CI performance. Gfeller et al. (2000) found that $83 \%$ of the CI users they surveyed reported diminished levels of music enjoyment after implantation when compared with their experiences before losing their hearing. Furthermore, 33\% stated that they avoided music because of its aversive sound. Gfeller et al. (2002) postulated that sound quality may contribute to overall appreciation more so than the ability to identify instruments, particularly for nonmusicians. Many of the published music appraisal studies involving CI users compared their ratings with those given by normally hearing subjects. In contrast, this study compared ratings from CI users with those from a group of hearing aid (HA) users who met the audiological criteria for a CI. In addition, to help account for between-subject variability, a third group of subjects who were on the waiting list (WL) for a CI participated in the study. Finally, because some of the published research has reported that increased complexity of musical stimuli can lead to lower ratings of pleasantness by CI users (Gfeller et al., 2003; Schulz \& Kerber, 1994), the present study also investigated whether the degree of complexity affected the subjects' ratings.

\section{Materials ANd Methods}

Fifteen CI subjects, 15 HA subjects, and nine WL subjects participated in this study. All subjects had a moderately-se $\equiv$ to profound hearing loss acquired postlingu ${ }_{y}$. The HA subjects were selected to meet the current CI criteria in terms of level of hearing loss and speech perception scores. All CI subjects used a Nucleus CI system (eight CI24 with the ACE processing strategy, and seven CI22 with the SPEAK strategy). The nine WL subjects were implanted with a Nucleus CI24 system and subsequently used the ACE strategy. The HA subjects, including the WL subjects when tested before implantation, used their own HA(s). The WL subjects were tested approximately 1 mo before implantation and 3 mo after activation of the CI. 
The assessments consisted of three subtests: 1) single instrument, 2) solo instruments accompanied by an orchestra, and 3) music ensembles. The stimuli for each subtest comprised four 5 -second extracts of music played by 12 different instruments or ensembles (i.e., 48 stimuli per test). The stimuli were obtained from commercially available compact disc recordings of real (i.e., not synthesized) sounds. The instruments for the first two subtests were: $=$ singer, female singer, piano, guitar, bass drun $=\mathrm{r}$ timpani), drum kit, xylophone, cello, violin, trumpet, flute, and clarinet. The ensembles in the third subtest were: $\equiv$ ir (four-part, a capella), orchestra, jazz band (in - mental), rock band (instrumental), country and western band (instrumental), string quartet, percussion ensemble, violin and piano duet, cello and piano duet, male singer and piano duet, female singer and piano duet, and a trio of one male and one female singer with piano accompaniment. For each musical extract, the researcher indicated which was playing from a list of the 12 instruments or ensembles. The subject was then asked to rate the pleasantness of each excerpt on a scale from 1 to 10 , where 1 was "very unpleasant" and 10 was "very pleasant." For the CI subjects, stimuli were presented using direct audio input. For the HA subjects, direct audio input via an audio shoe was used where available; otherwise, a neck-loop system was employed. Subjects used their preferred device settings, with stimuli presented at comfortable levels of loudness.

\section{Results}

A comparison of the mean ratings is provided in Figure 1 cepeated-measures two-way analysis of variance : conducted to compare the CI and HA groups' ratings across the three subtests. This showed no significant difference in ratings for the factor of group ( $p=0.386$ ) but a significant difference for the factor of subtests $(p<0.001)$. No significant interactions were found between the group and subtest factors ( $p=0.686$ ). The CI group generally provided higher ratings than the HA group for all three subtests, although the mean score differences were not statistically significant. The tests of the within-subjects contrasts showed significant differences between all three subtests (subtest 1 and 2: $p<0.001$; subtest 1 and $3: p<0.001$; subtest 2 and $3: p=0.014$ ), with appraisal scores being highest for subtest 1 and lowest for subtest 3 . For the WL subjects, a repeated-measures two-way analysis of variance showed that the postimplantation ratings were significantly higher than the preimplantation ratings $(p=0.026)$, along with a significant difference between the subtests' scores $(p=$ $0.034)$. There was no significant interaction between these two factors $(p=0.147)$. A test of within-subjects contrasts showed that the ratings for subtest 1 were significantly higher than those for subtest $3(p=$ 0.044). The postimplant ratings were not only higher than the preimplant ratings for all three subtests; they also were higher for all of the instruments or ensembles in each subtest.

The quality-rating assessment was part of a larger study that involved closed-set identification of the instruments or ensembles. Nonparametric Spearman's rho correlations were calculated to investigate the relationship between these quality ratings and the previously obtained identification scores for the $\mathrm{CI}$ and $\mathrm{H} \equiv$ groups. For both groups, the correlations were significant, but they were only moderate to weak (CI: rho = $0.325, p=0.029$; HA: rho $=0.491, p=0.001$ ).

\section{Discussion}

The results of this study suggest that although neither device enabled highly satisfactory music appreciation, the CI users judged music to sound more pleasant than equivalent HA users. This finding is somewhat surprising in consideration of our as-yet unpublished findings that HA users are better at musical pitch-perception tasks than CI users. There may be several explanations for this finding.

Fig. 1. A comparison of the average quality ratings for each subject group and subtest. Ratings were provided out of 10, with error bars indicating one standard deviation. *Statistically significant difference $(p<0.005)$.
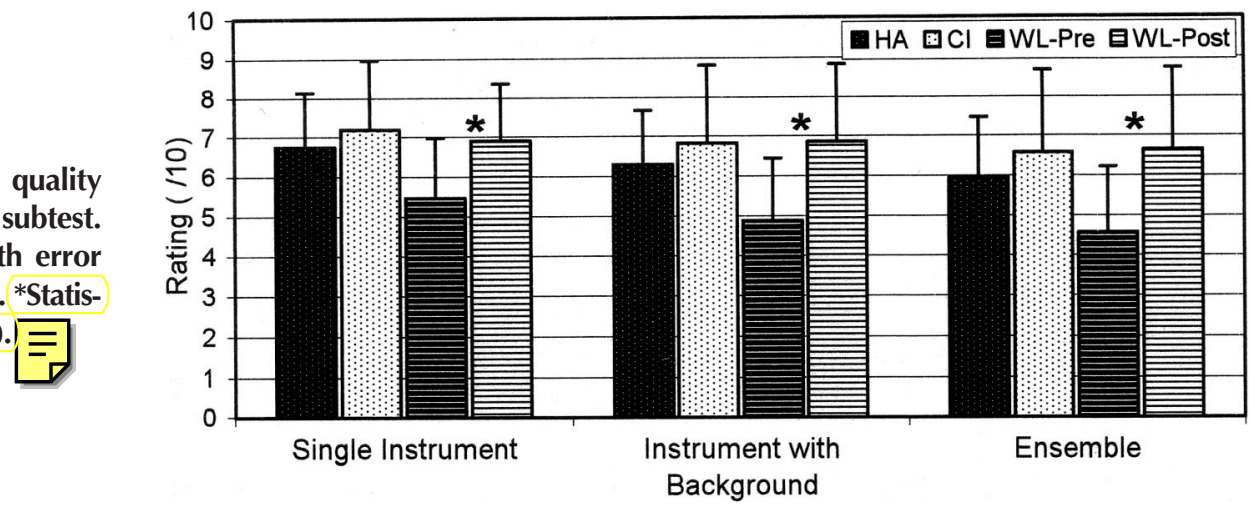
For CI recipients, although existing implant systems may not transmit sufficient spectral information to allow users to fully appreciate musical stimuli, the additional high-frequency information provided by the implant in comparison with the HA may have contributed to the perceived sound quality and timbre. For the HA subjects, the auditory filter anomalies associated with a significant cochlear hearing loss would probably have resulted in reduced frequency selectivity and spectral smearing. This would have restricted the amount of spectral detail perceived by the listener (Moore, 1995, 1996; Summers \& Leek, 1994).

On the other hand, the results may be attributable partly to personal bias and subjective views of the device. It is plausible that some CI users would assume that the implant is inherently a more sophisticated device than an $\mathrm{HA}$. This might have inflated the subjective rating $\equiv$ the present study. Despite this, comments by the newly implanted WL subjects support the significant difference between their ratings. Some of these subjects commented that they "got more" of the sound with the CI; when they had HAs, they only heard the beat or bass sounds, but with the implant, they could hear more of the higher-pitched melody instruments. Some described it as getting a "broader picture" of the musical sounds with more detail.

For all three subject groups, the highest mean ratings were obtained on the first subtest with the single instruments, whereas ratings were progressively lower for the second and third subtests. That is, music involving several instruments playing simultaneously was rated to sound less pleasant than music involving only single instruments. The weak to moderate strength of the correlations between identification scores and quality ratings for the experienced CI and HA users is not entirely surprising. After all, although these factors are somewhat related, the ability to identify an instrument or music group does not necessarily imply that one will like a musical work. Similarly, not knowing the artist or composer does not preclude enjoyment of a piece or song.

In conclusion, the music quality ratings of CI users in this study were generally higher than those of HA users with equivalent levels of hearing loss; this was particularly evident for the newer recipients of the device. Further, for all subject groups, single-instrument stimuli were preferred to music involving multiple instruments.

\section{ACKNOWLEDGMENTS}

Funding was provided by the National Health \& Medical Research Council of Australia, the CRC for Cochlear Implant \& Hearing Aid Innovation, and the Garnett Passe \& Rodney Williams Memorial Foundation.

Address for correspondence: Valerie Wei Looi, c/o Dept of Communication Disorders, The University of Canterbury, Private Bag 4800, Christchurch 8020, New Zealand.

Received $\bullet$; accepted $\bullet$.

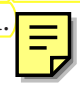

AQ: 2

\section{REFERENCES}

Gfeller, K., Christ, A., Knutson, J., Witt, S., \& Mehr, M. (2003). The effects of familiarity and complexity on appraisal of complex songs by cochlear implant recipients and normal hearing adults. Journal of Music Therapy, 40, 78-112.

Gfeller, K., Christ, A., Knutson, J. F., Witt, S., Murray, K. T., \& Tyler, R. S. Musical backgrounds, listening habits, and aesthetic enjoyment of adult cochlear implant recipients. (2000). Journal of the American Academy of Audiology, 11, 390-406.

Gfeller, K., Witt, S., Adamek, M., Mehr, M., Rogers, J., Stordahl, J., \& Ringgenberg, S. (2002). Effects of training on timbre recognition and appraisal by postlingually deafened cochlear implant recipients. Journal of the American Academy of Audiology, 13, 132-145.

Moore, B. C. J. (1995). Perceptual Consequences of Cochlear Damage. Oxford: Oxford University Press.

Moore, B. C. J. (1996). Perceptual consequences of cochlear hearing loss and their implications for the design of hearing aids. Ear and Hearing, 17, 133-161.

Schulz, E., \& Kerber, M. (1994). Music perception with the MED-EL implants. In I. J. Hochmair-Desoyer \& E. S. Hochmair (Eds.), Advances in Cochlear Implants (pp. 326-332). Vienna, Austria: Datenkonvertierung, Reproduktion und Druck.

Summers, V., \& Leek, M. R. (1994). The internal representation of spectral contrast in hearing-impaired listeners. Journal of the Acoustical Society of America, 95, 3518-3525. 


\section{AUTHOR QUERIES}

\section{AUTHOR PLEASE ANSWER ALL QUERIES}

AQ1: AUTHOR — Please verify locations for all affiliations.

AQ2: AUTHOR - Please verify correspondence address as shown and provide email address. AQ3: AUTHOR- Please define ACE and SPEAK at first use. 